\title{
Erfolge und Probleme der modernen (Mainstream-) Makroökonomik: Kommentar zum Beitrag von Rüdiger Bachmann
}

\section{Peter Spahn}

Online publiziert: 22. Februar 2019

(C) List-Gesellschaft e.V. 2019

Rüdiger Bachmann bearbeitet die ihm mit dem Titel des Beitrags gestellte Aufgabe, indem er - ähnlich wie Ricardo Reis (2018) - eine Vielzahl neuerer Arbeiten vorstellt, die einen detaillierten Eindruck vom Verlauf der gegenwärtigen Forschungsfront im Bereich der monetären Makroökonomie ${ }^{1}$ vermitteln. Die eher grundsätzlichen Debatten um eine „Krise der Makroökonomie“ sind für ihn eigentlich abgeschlossen; nur wenige ,,berechtigte“ Kritikpunkte bleiben übrig und werden am Schluss seines Beitrags kurz angesprochen.

Ein solches Vorgehen ist natürlich insbesondere für einen „Fan“ der modernen Makrotheorie völlig legitim, erschwert aber einen Kommentar. Angezeigt wäre eine Gegenprüfung der vorgestellten (und weiterer) Arbeiten: Wie wertvoll ist im Einzelnen der Erkenntnisfortschritt? Die Versuchung ist groß, die Trivialität des einen oder anderen Ergebnisses der modernen makrotheoretischen und -empirischen Forschung zu monieren: So seien individuelle Einkommensrisiken asymmetrisch, d.h. in Rezessionen sind ,große Einkommensverluste wahrscheinlicher, ohne dass die Wahrscheinlichkeit großer Einkommensgewinne zunimmt“; auch wurde nun entdeckt, ,dass Fiskalmultiplikatoren in Rezessionen größer sind als in konjunkturellen Normalzeiten oder Booms“. Beide Botschaften haben vermutlich für mit traditioneller Theorie ausgebildete VWL-Studenten kaum Neuigkeitswert.

\footnotetext{
1 Es geht also i.w.S. um den Themenbereich von Inflation, Beschäftigung und Makropolitik.
}

This commentary article refers to the article available online at https://doi.org/10.1007/s41025-01900121-y. An author's reply to this commentary article is available online at https://doi.org/10.1007/ s41025-019-00145-4.

\footnotetext{
P. Spahn $(\bowtie)$

Universität Hohenheim, Stuttgart, Deutschland

E-Mail: peter.spahn@uni-hohenheim.de
} 
Aber solch selektive Kritik wäre unfair; auch alle anderen Forschungsergebnisse wären eingehend zu würdigen. Dazu fehlt hier der Raum. Auch aus einem wichtigen anderen Grund richtet sich dieser Kommentar doch wieder auf die allgemeinere Ebene: Die moderne Neukeynesianische Makroökonomie (NKM) steht nach wie vor nicht so sehr wegen ihrer Detailforschung, sondern wegen der wenig überzeugenden Konstruktion ihres Grundmodells in der Kritik. ${ }^{2}$ Zwar besagt ein altes Forschungsprinzip, dass Modelle nicht realistisch sein müssen, sondern gute Prognosen ermöglichen sollen (Friedman 1953) - aber sie sollen doch auch etwas erklären. Selbst wenn es gelänge, einen Zusammenhang zwischen Storchenflug und Geburtenrate durch definitorische Kunstgriffe und immer perfektere statistische Methoden zu untermauern, so bliebe doch die Vermittlung des gesamten Ansatzes eine heikle Aufgabe.

Die NKM ist (wie auch die Theorie der Real Business Cycles als ihr Vorläufer) intuitiv wenig überzeugend. Das hat nichts mit dem verwendeten Gleichungssystem zu tun. Für die unterstellten Erwartungsstrukturen und möglichen Time Lags in Angebots- und Nachfragefunktionen ließen sich zahlreiche mikro- und makroökonomische Begründungen finden, und die aktive zinspolitische Regel stellt einen großen Fortschritt gegenüber dem IS-LM-Modell dar. ${ }^{3}$ Das Problem ist die Story hinter diesen Gleichungen. Diese entstammt (wie Bachmann unterstreicht) der Gedankenwelt der optimalen Wachstumstheorie, die eine Robinson-Crusoe-Wirtschaft oder ein von einem Social Planner gelenktes Kollektiv beschreibt. Ein repräsentativer Akteur plant und realisiert (bei exogenen Schocks) einen optimalen Pfad von Arbeit, Konsum und Sparen über die Zeit. Nicht nur Studenten widerstrebt es, in diesem Bild dem Kern unserer Marktwirtschaft zu erkennen.

Natürlich ist das Grundmodell mittlerweile vielfach ausdifferenziert worden. Aber dadurch kann man seinen Defekten nicht entkommen. Für den zentralen Baustein einer zinsabhängigen Verschiebung des Konsums auf der Zeitachse gibt es wenig Evidenz. ${ }^{4}$ Die Einkommensbildung durch die Investition spielt keine große Rolle. Die Beschäftigung erscheint im Modell (wie für die Wachstumstheorie typisch) angebotsbedingt; sie wird zwar auch von Nachfrageschocks beeinflusst, aber in Übereinstimmung mit den Präferenzen angepasst: „Movements take place along a labor supply curve“ (Blanchard 2008, S. 12). Unfreiwillige Unterbeschäftigung widerspricht der Konzeption, sie wird als Sucharbeitslosigkeit gedeutet. Wohlfahrtstheoretische und wirtschaftspolitische Schlussfolgerungen aus derartigen Modellen sind daher mit Vorsicht zu behandeln.

In modernen Mehrsektorenmodellen finden sich heterogene Akteursgruppen mit unterschiedlichen Zeitpräferenzen sowie Banken, die als Intermediäre zwischen Sparern und Investoren vermitteln. Aber es ist eben kein Zufall, dass dabei die

\footnotetext{
2 Argumente dazu finden sich u.a. bei Woodford (2011), Korinek (2015), Romer (2016), Blanchard (2018), Krugman (2018), Lavoie (2018) und Wren-Lewis (2018). Zu einer theoriegeschichtlich breiter angelegten Perspektive siehe Spahn (2016).

3 In der Lehre ist dieses sog. Drei-Gleichungs-System sehr hilfreich und leistungsfähig (siehe z. B. Carlin und Soskice 2006 oder Spahn 2012).

4 ,The basic Euler equation model is at odds with the data, in the sense that consumption growth does not react to the real interest rate and appears to be backward-looking“" (Ascari et al. 2016, S. 19).
} 
alte verquere Sichtweise propagiert wird, wonach die Bank zuvor eingesammelte Einlagen von Sparern an die Kreditnehmer weiterverleiht. Die Konsequenz dieses falschen Bankverständnisses ist, dass die gesamtwirtschaftliche Dynamik in Modellsimulationen systematisch verzerrt dargestellt wird (Jakab und Kumhof 2015). Die NKM sträubt sich aber dagegen, die mit Bilanzverlängerung einhergehende Kreditschöpfung der Banken in den Mittelpunkt zu stellen, weil dabei die beschworene Führungsrolle des optimierenden Haushalts verloren ginge. Die Prägung des wissenschaftlichen Denkens durch ein unangemessenes Weltbild geht bei einigen Ökonomen (z. B. Reis 2013) so weit, konsequenterweise auch die Geldschöpfungsfähigkeit der Notenbanken zu ignorieren. Diese werden als liquiditätsbeschränkte Akteure dargestellt, die sich zunächst Finanzmittel am Geld- und Kapitalmarkt beschaffen müssen, um damit z.B. staatliche Wertpapiere kaufen zu können. ${ }^{5}$

Abgesehen von solchen partiellen Ungereimtheiten steckt die NKM mit dem Dogma der „Mikrofundierung der Makroökonomie“ in einem selbstgewählten, unlösbaren Dilemma. Einerseits ist das gesamtwirtschaftliche Geschehen unter Beachtung aller Rückwirkungen letztlich ein Ergebnis einzelwirtschaftlicher Entscheidungen auf der Mikroebene, folglich könnte man versuchen, daraus direkt die makrotheoretischen Beziehungen abzuleiten. Andererseits scheitert eine Analyse der interdependenten Optimierung von $n$ Akteuren über $m$ Güter und alle künftigen Perioden an der übergroßen Komplexität. Ein Robinson-Crusoe-Modell ist insofern genial, als es diesem Dilemma entgeht: Mikro- und Makroökonomie fallen zusammen. Aber mit jedem Schritt zu mehr Heterogenität und sektoraler Auffächerung verschärft sich das Problem wieder.

Die formale Unhandlichkeit der NK-Modelle erschwert nicht nur die wissenschaftliche Kommunikation, sondern erzeugt substanzielle Probleme:

1. Da die quantitative Erforschung von Modellzusammenhängen nicht über die ökonometrische Schätzung einzelner Gleichungen, sondern des gesamten Modells erfolgt, fehlt gerade bei umfangreichen Systemen oft eine empirische Basis zur Bestimmung aller Modellparameter. Man behilft sich dann, indem einige Parameter „kalibriert“, d.h. so gesetzt werden, dass das Modell den typischen Konjunkturverlauf möglichst gut trifft. Das ist nahe am Etikettenschwindel. Auch „tiefe“ Modellbausteine wie die verwendeten Nutzenfunktionen werden im Nachhinein so modifiziert, dass die Simulationen den makroökonomischen Fakten entsprechen. Das ist eher eine Makrofundierung der Mikroökonomie.

2. Die standardmäßig unterstellten rationalen Erwartungen sind in komplexen Modellen faktisch nicht bestimmbar. Im einfachen Zwei-Gleichungs-Ansatz der Neuklassik (bestehend aus einer Phillips-Kurve und einer quantitätstheoretisch motivierten Nachfragefunktion) war diese Erwartungshypothese noch plausibel. Allgemein ist zur Berechnung rationaler Erwartungen - nach McCallums (1998) Methode der unbestimmten Koeffizienten - eine Vorabkenntnis der qualitativen Funktionsmechanismen des Modells bzw. der Wirtschaft nötig. Treten dabei neben Erwartungen

\footnotetext{
5 Ein hochrangiger Vertreter dieser Ansicht erläuterte dies in einer privaten Kommunikation so: ,The goal is to, as much as possible, describe central banks the same way we do any other economic agent. Thus taking away the mystique. When an economic agent buys new assets, we talk of the liabilities it raises to fund those purchases".
} 
für die folgende Periode noch Time Lags auf, so sind bei $x$ endogenen Variablen Matrizen der Dimension $x^{2} \mathrm{zu}$ bearbeiten.

Für die Ökonomen mit ihrer Matlab-Software ist das alles kein Problem. Aber rationale Erwartungen bedeuten ja, dass nicht die Wissenschaftler, sondern die Marktakteure die Lösung des Modells berechnen und auf dieser Grundlage ihre Erwartungen bilden. Bei mehr als zwei Variablen ist dies illusorisch. Die Wirtschaftsbürger sind wohl eher nicht in der Lage, Makromodelle per Forward Solving zur Erwartungsbildung zu nutzen. Auch lerntheoretisch modifizierte rationale Erwartungen setzen aber voraus, dass die Akteure über ein qualitativ weitgehend korrektes Abbild des gesamten Wirtschaftsablaufs verfügen. Die Erwartungsterme in komplexen NK-Modellen sind also praktisch leer; begründbar und realistisch wären adaptive Erwartungen.

3. Mikrofundierte NK-Modelle erliegen einer „Tyrannei der Vollständigkeit“ eine Folge ihres Charakters als allgemeiner Gleichgewichtstheorie, die korrekterweise sämtliche Interdependenzen zwischen allen Akteuren und Variablen im Blick hat, und des unterstellten Optimierungsverhaltens, das den Leuten Planungen bis ins Unendliche abverlangt. So berücksichtigen die Haushalte im Modell bei ihren Entscheidungen u. a. die Konsequenzen aus den zu erwartenden Veränderungen der langfristigen Verschuldungsposition des Staates, die sie ebenso wie die Regierung natürlich berechnen können. Daraus folgt dann zwingend, dass etwa konjunkturpolitisch gemeinte Steuersenkungen unausweichlich durch Mehrersparnis kompensiert werden, weil die Haushalte ja wissen, dass die Regierung zur Konstanthaltung ihres Schuldenstandes irgendwann in der Zukunft die Steuern erhöhen wird. Das ist die berühmte ,ricardianische Äquivalenzthese“, die schon länger beschworen wird und auch im Rahmen der NKM nicht fehlen darf. Es spielt keine Rolle, dass die beschriebene Haushaltsreaktion selten beobachtet wurde - sie erscheint logisch zwingend. ${ }^{6}$

Infolge der „Tyrannei der Vollständigkeit“ gelten extreme Fernwirkungen heutiger Ereignisse als Standardfall, der aber faktisch unrealistisch ist. Man kann dieser verzerrenden Modellphilosophie aber kaum entkommen. Von der Optimierungsannahme abzuweichen (,Alles nach Periode $t+7$ spielt keine Rolle mehr") wäre beliebig. Alternativ dazu könnte man „Friktionen“ einbauen, Unvollkommenheiten in Märkten und Institutionen, aber diese wären ja ebenfalls zu begründen. Vor allem würde dies die Komplexität des Modells noch weiter erhöhen. Daher ist reine Optimierung die Regelannahme; das ist leichter zu berechnen. ${ }^{7}$ Imperfektionen können nur partiell zugelassen werden, um die Analyse formal beherrschbar zu halten. Daher haben die NK-Modelle einen Bias zugunsten vollständiger und glatt funktionierender Systeme. Der grundlegende Optimierungsapparat, die Ableitung der EulerGleichungen, wird mit größter Sorgfalt zelebriert, für die gleichzeitige Analyse von wichtigen „Friktionen“ (z. B. realistische Erwartungs- und Lernstrukturen, Endoge-

\footnotetext{
6 Tatsächlich könnte die Regierung durchaus auch anders handeln und die langfristige Zins-WachstumsRelation zur Richtschnur wählen bzw. zu einem anderen stabilen oder instabilen (!) Verschuldungsstand übergehen.

7 „Models of rational behavior became standard because they were the easiest to solve“ (Thaler 2016, S. 1579).
} 
nität der Natural Rates von Produktion und Beschäftigung, eine vom Sparverhalten unabhängige Bankaktivität) fehlt dann der Raum. ${ }^{8}$

Auch dieser Kommentar schließt sich daher der von prominenten Ökonomen geforderten (zumindest ergänzenden) ,Zulassung“ von reduzierten Makromodellen ohne explizite Mikrofundierung an. Das oft präsentierte Gegenargument der LucasKritik kann nicht wirklich überzeugen. Zum einen ist die propagierte Aufteilung von Modellmechanismen und -parametern in „tiefe“ Strukturkonstanten einerseits und politikabhängige Elemente andererseits (wie auch Bachmann andeutet) nicht strikt durchzuhalten; warum soll nicht auch die Zeitpräferenz der Akteure auf Politikmaßnahmen reagieren? Zum anderen ist das Bild von den Wirtschaftssubjekten, die mit eindeutigen rationalen Erwartungen politische Aktionen durchkreuzen, wie bereits erwähnt fern der Realität.

Die Frage ist, wozu man Makrotheorie braucht. Wenn es z. B. um die Analyse von Funktionsproblemen der Eurozone in den 2000er Jahren geht (Realzins-, Konjunktur- und Leistungsbilanzdivergenzen), um den Zusammenhang zwischen privater Überschuldung in Peripherieländern und Verlust ihrer Wettbewerbsfähigkeit oder um die konjunkturellen Konsequenzen einer an variablen Sicherheiten orientierten Bankkreditvergabe (Spahn 2015), reichen reduzierte Modelle mit angedeuteter Mikrofundierung à la $I S-L M$ völlig aus. Es gibt sicher auch spezielle Fragestellungen, bei denen die Herleitung von gesamtwirtschaftlichen Marktbeziehungen aus individuellen Nutzenfunktionen sinnvoll sein kann - aber die oben genannten Einschränkungen bleiben bestehen.

Helfen die pluralistischen Ansätze weiter? Zunächst ist zu bemerken, dass Kampagnen zur Kritik der ,herrschenden“ Wirtschaftstheorie und Forderungen nach ihrer paradigmatischen Erweiterung nicht neu sind; häufig beruhen die Streitpunkte auf Missverständnissen seitens der Kritiker. So wurde der Terminus „Gleichgewichtstheorie" immer wieder so gedeutet, als ob Koordinationsmängel und Wirtschaftskrisen ausgeschlossen würden.

Ebenso alt ist die Kritik an der Figur des Homo Oeconomicus: Neue Erkenntnisse der Psychologie würden darauf hinweisen, dass die Menschen keineswegs stets in rationaler Weise ihren Nutzen maximieren. Dabei wird jedoch übersehen, dass die Volkswirtschaftslehre spätestens seit der Ausdifferenzierung der Sozialwissenschaften zu Beginn des 20. Jahrhunderts primär nicht das Verhalten des Menschen, sondern die Funktionsweise von Märkten und der Marktinteraktion erklären will. Die Modelle werden von künstlichen „Akteuren“ bewegt, die ihre Entscheidungsenergie aus simplen Regeln beziehen. Der zu maximierende „Nutzen“ kann, muss aber nicht ausschließlich durch Gütermengen definiert werden. Die „Rationalität“ der Handlungen ist immer in Bezug zu Entscheidungsrestriktionen zu setzen, die natürlich auch aus Grenzen der Informationsverfügbarkeit oder Denkfähigkeit resultieren können. Das „Bauchgefühl“, nach dem die Menschen angeblich entscheiden, darf nicht als realistischere Verhaltenstheorie gegen die neoklassische Optimierungslogik

\footnotetext{
8 ,DSGE [...] has made the wrong modelling choices, choosing complexity in areas where the core story of macroeconomic fluctuations could be told using simpler hypotheses, but simplifying in areas where much of the macroeconomic action takes place“" (Stiglitz 2018, S. $74 \mathrm{f}$.).
} 
ausgespielt werden; letztere übersetzt die faktischen Verhaltensmuster nur in eine analytische Sprache. ${ }^{9}$

Der formale Ansatz ist damit hinreichend tautologisch, um für vielfältige Fragestellungen anwendbar zu sein. Einer behavioristischen Erweiterung insbesondere der Makrotheorie bedarf es insofern nicht. ${ }^{10}$ Aber hier ist eine wichtige Einschränkung angebracht. Die Makrotheorie hat sich wie bereits erwähnt mit der Hypothese rationaler, d.h. modellkonsistenter Erwartungen in ein Dilemma manövriert, indem sie die Aussagen der Theorie daran bindet, dass die Marktakteure an eben diese Theorie glauben. Kein ernstzunehmender Theoretiker kann aber heute noch davon ausgehen, dass eine einzige Theorie die Deutungshoheit in einer Marktgesellschaft besitzt. ${ }^{11}$ Die Erwartungsbildung der Wirtschaftssubjekte ist deshalb zu Recht ein zentrales Thema der modernen Makrotheorie. Dabei geht es nicht nur wie in den frühen Ansätzen zum Macroeconomic Learning um die Analyse der Bedingungen, die den Akteuren die Abschätzung quantitativer Beziehungen innerhalb eines für „wahr" befundenen Modells erlauben; wichtiger ist, ob und wie sie sich zwischen alternativen theoretischen Weltbildern (in soziologischem Jargon: Deutungsmustern) entscheiden. Neben nutzentheoretisch gestützten und makroökonomisch reduzierten Modellen können in diesem Forschungsfeld auch behavioristische Ansätze eine wichtige Rolle spielen. ${ }^{12}$

Rüdiger Bachmann schreibt, die Makroökonomik sei heute soziologisch divers und plural ausgerichtet wie nie zuvor. Pluralistische Kritiker werden diese Einschätzung vermutlich bestreiten. Aber dadurch sollte sich die moderne Makrotheorie nicht in die Defensive drängen lassen. Man muss der populistischen Forderung nach mehr Interdisziplinarität nicht unbedingt nachgeben und sollte auf den komparativen Vorteilen des modelltheoretischen Instrumentariums der Ökonomie bestehen. Es ist fraglich, ob etwa die neuroökonomische Erforschung der Gehirnvorgänge bei Anlageentscheidungen dem Fortschritt der ökonomischen Theorie dient; von den wirklichen Ursachen der Finanzkrise lenkt dies nur ab. Die makroökonomischen Modelle sollten in knapper Form auf die jeweils im Fokus stehenden Fragestellungen ausgerichtet und nicht in jedem Fall mit nutzentheoretischer Propädeutik verkompliziert werden.

\footnotetext{
9 Joan Robinson (1965 [1962], S. 111) macht sehr schön deutlich, dass es dabei eben nicht um einen Gegensatz geht: „Marshall beschreibt einen Jungen, der Brombeeren pflückt und das solange tut, bis der Grenznutzen einer weiteren Beere nicht mehr ausreicht, um das Grenzleid einer zusätzlichen Anstrengung aufzuwiegen. - Kurz, er hört auf, wenn er nicht mehr mag“.

10 „Behavioral economics is not needed to explain macroeconomic puzzles. The standard optimizing framework can be very expansive and elastic - it may be possible to find a ,non-behavioral ${ }^{\circ}$ optimizing explanation for every apparent fault in the standard New Keynesian models" (Driscoll und Holden 2014, S. 143; vgl. auch Spahn 2016, S. 185 ff.).

${ }_{11}$ Sargent selbst beschrieb seine Theorie mit ironischem Unterton so: „All agents inside the model, the econometrician, and God share the same model“ (in Evans und Honkapohja 2005, S. 566; vgl. auch Riese 1983).

12 Zu einem Überblick siehe De Grauwe (2012), Woodford (2013), Eusepi und Preston (2016) und Gabaix (2016).
} 


\section{Literatur}

Ascari, G., et al. (2016). Empirical evidence on the euler equation for consumption and output in the US. Oxford: University of Oxford.

Blanchard, O. J. (2008). The state of macro. NBER Working Papers, 14259. Cambridge: National Bureau of Economic Research.

Blanchard, O.J. (2018). On the future of macroeconomic models. Oxford Review of Economic Policy, 34(1-2), 43-54.

Carlin, W., \& Soskice, D. (2006). Macroeconomics—imperfections, institutions and policies. Oxford: Oxford University Press.

De Grauwe, P. (2012). Lectures on behavioral macroeconomics. Princeton, Woodstock: Princeton University Press.

Driscoll, J.C., \& Holden, S. (2014). Behavioral economics and macroeconomic models. Journal of Macroeconomics, 41, 133-147.

Eusepi, S., \& Preston, B. (2016). The science of monetary policy-an imperfect knowledge perspective. Federal Reserve Bank of New York Staff Reports, Bd. 782.

Evans, G. W., \& Honkapohja, S. (2005). An Interview with Thomas J. Sargent. Macroeconomic Dynamics, 9, 561-583.

Friedman, M. (1953). The methodology of positive economics. In M. Friedman (Hrsg.), Essays in Positive Economics (S. 3-43). Chicago: University of Chicago Press.

Gabaix, X. (2016). A behavioral new Keynesian model. CEPR Discussion Papers, Bd. 1729. London: Centre for Economic Policy Research.

Jakab, Z., \& Kumhof, M. (2015). Banks are not intermediaries of loanable funds-and why this matters. Bank of England, working papers, Bd. 529.

Korinek, A. (2015). Thoughts on DSGE macroeconomics-matching the moment, but missing the point? Baltimore: Johns Hopkins University.

Krugman, P.R. (2018). Good enough for government work? Macroeconomics since the crisis. Oxford Review of Economic Policy, 34(1-2), 156-168.

Lavoie, M. (2018). Rethinking macroeconomic theory before the next crisis. Review of Keynesian Economics, 6, 1-21.

McCallum, B. T. (1998). Solutions to linear rational expectations models. Economic Letters, 61, $143-147$.

Reis, R. (2013). The mystique surrounding the central bank's balance sheet. American Economic Review, Papers and Proceedings, 103, 135-140.

Reis, R. (2018). Is something really wrong with macroeconomics? Oxford Review of Economic Policy, 34(1-2), 132-155.

Riese, H. (1983). Zur Theorie rationaler Erwartungen - Neoklassische Grundlage und keynesianischer Gegenentwurf. In G. Bombach \& : (Hrsg.), Makroökonomik heute - Gemeinsamkeiten und Gegensätze (S. 249-277). Tübingen: Mohr Siebeck.

Robinson, J. (1965). Doktrinen der Wirtschaftswissenschaft. München: C. H. Beck.

Romer, P. M. (2016). The trouble with macroeconomics. New York: Stern School of Business, New York University.

Spahn, P. (2012). Geldpolitik- Finanzmärkte, neue Makroökonomie und zinspolitische Strategien (3. Aufl.). München: Beck.

Spahn, P. (2015). The bank lending channel with endogenous money-a simple macro model. Credit and Capital Markets, 48, 565-593.

Spahn, P. (2016). Streit um die Makroökonomie - Theoriegeschichtliche Debatten von Wicksell bis Woodford. Marburg: Metropolis.

Stiglitz, J.E. (2018). Where modern macroeconomics went wrong. Oxford Review of Economic Policy, 34(1-2), 70-106.

Thaler, R.H. (2016). Behavioral economics_-past, present, and future. American Economic Review, 106, 1577-1600.

Woodford, M. (2011). What's wrong with economic models?. New York: Columbia University.

Woodford, M. (2013). Macroeconomic analysis without the rational expectations hypothesis. NBER Working Papers, Bd. 19368. Cambridge: National Bureau of Economic Research.

Wren-Lewis, S. (2018). Ending the microfoundations hegemony. Oxford Review of Economic Policy, 34(1-2), 55-69. 